\title{
Effects of PTEN on the proliferation and apoptosis of colorectal cancer cells via the phosphoinositol-3-kinase/Akt pathway
}

\author{
YAN SUN $^{1 *}$, HUA TIAN $^{2 *}$ and LIN WANG ${ }^{3}$ \\ ${ }^{1}$ Department of Gastroenterology, The Third Affiliated Hospital of Guangzhou Medical University, \\ Guangzhou, Guangdong 510150; ${ }^{2}$ Department of Gastroenterology, Houjie Hospital Affiliated \\ to Guangdong Medical College, Dongguan, Guangdong 523900; ${ }^{3}$ Department of Oncology, \\ Guangzhou Red Cross Hospital, Guangzhou, Guangdong 510150, P.R. China
}

Received September 4, 2014; Accepted December 5, 2014

DOI: $10.3892 /$ or.2015.3804

\begin{abstract}
Colorectal cancer (CRC) is one of the most common type of malignancy with a poor prognosis, due to a high frequency of metastasis and tumor recurrence. It has been reported that deletion and/or mutation of the PTEN gene can be involved in the pathogenesis of many types of cancers through the activation of the PI3K/Akt signaling pathway. Immunohistochemical staining was conducted to detect PTEN expression in CRC, adenomas and normal tissues. For the measurement of cell proliferation, CCK-8 was used. Apoptotic cells were quantified using FACS. Immunohistochemical staining results demonstrated that the expression of PTEN gradually decreased from normal colorectal mucosa, to colon hyperplastic polyps, adenomas, and ultimately primary colorectal adenocarcinomas. Upregulation of PTEN expression inhibited the proliferation of LoVo and SW480 cells, inducing G1 phase arrest and reducing the number of cells in the S phase. LoVo and SW480 cells with upregulated PTEN were sensitive to apoptosis induced by 5-FU. In addition, upregulation of PTEN inhibited the activity of Akt and activated the FoxO transcription factor. This is the first report of a gradual decrease in expression of PTEN from normal colon epithelial tissue to colon hyperplastic polyps, colorectal adenomas and finally CRC. Upregulation of PTEN inhibited the activity of the Akt pathway and regulated downstream genes involved in the cell cycle. These results suggest that inhibition of CRC cell proliferation and cell cycle arrest by PTEN are closely related to PI3K/Akt/FoxO.
\end{abstract}

Correspondence to: Dr Yan Sun, Department of Gastroenterology, The Third Affiliated Hospital of Guangzhou Medical University, Guangzhou, Guangdong 510150, P.R. China

E-mail: sunyansci@163.com

Dr Hua Tian, Department of Gastroenterology, Houjie Hospital Affiliated to Guangdong Medical College, Dongguan, Guangdong 523900, P.R. China

E-mail:1myh720@126.com

${ }^{*}$ Contributed equally

Key words: PTEN, proliferation, apoptosis, colorectal cancer cells, PI3K

\section{Introduction}

Colorectal cancer (CRC) is one of the world's most common type of malignancy, with more than 1.2 million cases occurring every year. The prognosis of CRC is poor due to frequent metastasis and tumor recurrence. Worldwide, approximately one million new cases of CRC occur annually, amounting to 492,000 related deaths (1). With many changes in the diet and lifestyle of individuals, CRC has become the third most common type of digestive tumor in China, and the number of new cases is increasing each year (2). The overall incidence is identical in men and women, with the risk beginning at age 40 and increasing with age. Thus, CRC ranks among the most frequent causes of cancer-related deaths in China. Despite improvements in surgery, radiotherapy and chemotherapy, unfortunately, the prognosis for CRC has not improved over the past decades (3), with an overall 5-year survival rate of approximately 40-50\%. Therefore, novel diagnostic tools and treatments need to be developed.

Phosphatidylinositol-3-kinase (PI3K) is a lipid kinase and is responsible for the phosphorylation of position 3 of the inositol ring of PI $(4,5) \mathrm{P} 2$. This generates the second-messenger PI (3-5) P3, which is required for fundamental cellular functions including transcription, translation, proliferation, growth and survival. Abnormal activation of PI3K signaling not only leads to neoplastic transformation of normal cells, but also correlates with tumor cell migration, adhesion, angiogenesis and degradation of the extracellular matrix (4). Phosphatase and tensin homolog deleted on chromosome ten (PTEN) is a tumor-suppressor gene located on human chromosome 10q23.3. PTEN is both a lipid phosphatase and a protein phosphatase. As a lipid phosphatase, PTEN classically converts phosphatidylinositol-3,4,5-trisphosphate (PIP3) to phosphatidylinositol-4,5-bisphosphate (PIP2) in the cytoplasm, thereby directly antagonizing the activity of PI3 kinase (PI3K) (5). Inactivation of PTEN results in constitutive activation of the PI3K/AKT pathway and therefore increases protein synthesis, proliferation, migration and survival (6). It has been reported that deletion and mutation of PTEN are involved in the pathogenesis of cancer by activating the PI3K/Akt signaling pathway (7).

Forkhead box $\mathrm{O}$ (FoxO) transcription factors belong to the Forkhead family of proteins, a family characterized by 
a conserved DNA binding domain termed the Forkhead box (Fox). These transcription factors are downstream targets of the serine/threonine protein kinase B (PKB)/Akt (8). Phosphorylation of FoxOs by Akt inhibits the transcription function of FoxOs and contributes to cell survival, growth and proliferation (9). FoxOs can promote cell growth inhibition and/or apoptosis signaling by inducing expression of multiple pro-apoptotic members of the $\mathrm{Bcl} 2$ family, stimulating expression of death receptor ligands, or by enhancing levels of various cyclin-dependent kinase inhibitors (CDKIs) (10). Coupled with their ability to cross-talk with p53, FoxOs represent an important class of tumor suppressors in a variety of cancers.

At present, the relationship between biological changes resulting from changes in PTEN expression and the FoxO transcription factor is unknown. In this study, both the expression and significance of PTEN function in the progression of CRCs were investigated. CRC cell lines LoVo and SW480 overexpressing PTEN were constructed to investigate the effects of PTEN expression on CRC cell proliferation, cell cycle and cell apoptosis. Proteins involved in the PI3K signaling pathway including Akt, FoxO transcription factors and proteins associated with the cell cycle were also investigated to elucidate the molecular mechanisms behind tumor proliferation and apoptosis.

\section{Materials and methods}

Specimens. Thirty-nine cases of colorectal adenomas and hyperplastic polyp specimens were collected from August 2007 to September 2009 at Nanfang Hospital, Southern Medical University, Guangzhou, China. Among these 39 cases, 22 males and 17 females were enrolled, ranging in age from 20 to 75 years with an average age of 46.3. Forty-five cases of colorectal cancer (CRC) and adjacent normal tissues (at least $10 \mathrm{~cm}$ away from the tumor) were obtained simultaneously from the patients at Nanfang Hospital. Among these 45 cases, 27 males and 18 females were enrolled, ranging in age from 32 to 83 years with an average age of 56. All of these cases were pathologically confirmed post-surgery. According to the criteria of the International Colorectal Cancer Collaborative Group, 6 cases were categorized as colorectal cancer Dukes' A stage. Thirteen cases, 20 cases, and 6 cases belonged to Dukes' B stage, Dukes' C stage, and Dukes' D stage, respectively.

Cell lines. CRC cell lines SW480 and LoVo were purchased from the American Tissue Culture Collection (ATCC) and were provided by the Shanghai Institute of Cell Biology, Chinese Academy of Sciences. LoVo cells were derived from a left supraclavicular lymph node metastases of a 56-year-old male colon adenocarcinoma patient. SW480 cells were derived from the primary tumor site of a 51-year-old male patient with Duke's B stage colon cancer.

Cell transfection. The plasmid pc-DNA3.1 (+) containing a CMV promoter and a neomycin selection marker was purchased from Invitrogen. Plasmid pc-DNA3.1-PTEN was a kind gift from Dr Guiqin Hou (Cellular Biology Laboratory of Zhengzhou University). LoVo and SW480 cells were transfected with pc-DNA3.1-PTEN or the control plasmid
pcDNA3.1 (Gibco Invitrogen, Grand Island, NY, USA) using the Lipofectamine $2000^{\mathrm{TM}}$ transfection reagent (Invitrogen). For stable cell population selection, at $24 \mathrm{~h}$ post-transfection, cells were re-plated in RPMI-1640 (Gibco BRL, Rockville, MD, USA) with $10 \%$ (vol/vol) fetal calf serum (FCS) and $1000 \mu \mathrm{g} / \mathrm{ml}$ G418 (Sigma, St. Louis, MO, USA). G418-resistant clones were selected and expanded. The mRNA and protein levels of PTEN in these cells were analyzed by RT-PCR and Western blot analysis. Of note, cells transfected with the control plasmid pc-DNA3.1 were cultured under the same conditions.

Isolation of total RNA and RT-PCR. Total RNA was isolated using Trizol reagent (Invitrogen Life Tech) according to the manufacturer's instructions. Total RNA $(3 \mu \mathrm{g})$ was reverse transcribed into single-stranded cDNA using RevertAid First Strand cDNA Synthesis kit (Fermentas). cDNA was amplified with forward (F) and reverse (R) primers by PCR as described below. The primer sequences for GAPDH were 5'-CGGG AAGCTTGTCATCAATGG-3' (F) and 5'-GGCAGTGAT GGCATGGACTG-3' (R). The primer sequences for PETN were 5'-GGAAAGGGACGAACTGGTGT-3' (F) and 5'-CAG GTAACGGCTGAGGGAAC-3' (R). Primers were used at a final concentration of $1 \mu \mathrm{M}$. Reaction mixture was first denatured at $94^{\circ} \mathrm{C}$ for $5 \mathrm{~min}$. The PCR was performed for 30 cycles of $94^{\circ} \mathrm{C}$ for $45 \mathrm{sec}, 58^{\circ} \mathrm{C}$ for 45 seconds, and $72^{\circ} \mathrm{C}$ for $45 \mathrm{sec}$, followed by $72^{\circ} \mathrm{C}$ for $10 \mathrm{~min}$. PCR products were analyzed using an agarose gel and visualized by ethidium bromide staining.

Western blotting. Nuclear and cytoplasmic protein was extracted using a Nuclear and Cytoplasmic Protein Extraction kit (Thermo Scientific, USA). Cells were harvested and lysed in RIPA buffer (RIPA kit, Shanghai Shenneng Bocai Biotechnology Co., Shanghai, China). The supernatant was collected by centrifugation for $20 \mathrm{~min}$ at $4^{\circ} \mathrm{C}$. The extracted proteins $(20 \mu \mathrm{g})$ were resolved on a $10 \%$ SDS-PAGE gel, and transferred to polyvinylidene difluoride Immobilon membranes. The membranes were blocked with 5\% non-fat milk and incubated with the appropriate primary antibody. After washing, the membranes were stained with the secondary antibody. Protein bands were visualized by chemiluminescent detection (ECL) (Amersham Biosciences).

Immunohistochemical staining to detect PTEN expression in CRC, adenomas and normal tissues. Paraffin-embedded tissues were sectioned $(4-\mu \mathrm{m})$ and placed onto glass slides. The slides were dehydrated and antigen was retrieved in $1 \%$ citric acid solution under microwave conditions. The slides were blocked with serum, followed by treatment with a catalase inhibitor for $10 \mathrm{~min}$ at room temperature. The slides were then incubated with the PTEN antibody (1:100) in a humidified chamber at $4^{\circ} \mathrm{C}$ for $12 \mathrm{~h}$. Slides were washed 3 times with PBS and stained with a biotin-labeled secondary antibody for $40 \mathrm{~min}$, followed by a streptavidin peroxidase solution for $30 \mathrm{~min}$ at room temperature after 3 washes with PBS. The slides were developed using DAB staining solution for $5 \mathrm{~min}$ followed by hematoxylin for $10 \mathrm{sec}$.

The intensity of staining was determined as described previously (43). No staining, light yellow staining, yellow staining, and brown staining were marked as $0,1,2$ and 


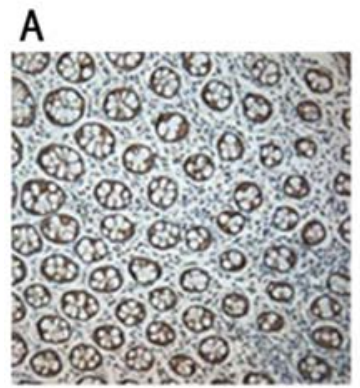

D
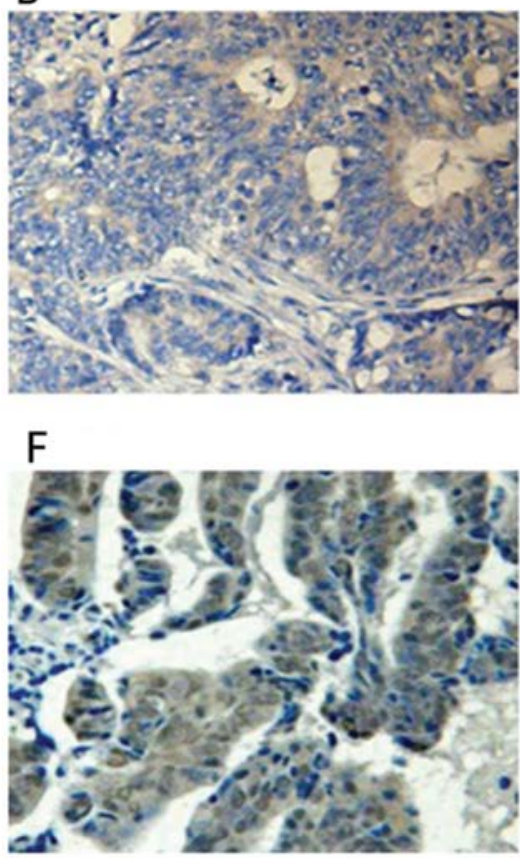

B

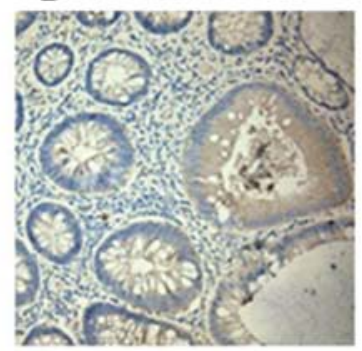

C

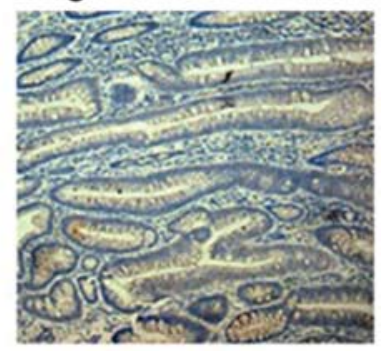

E

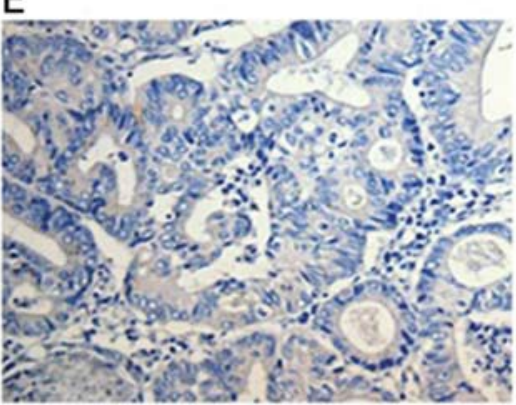

G

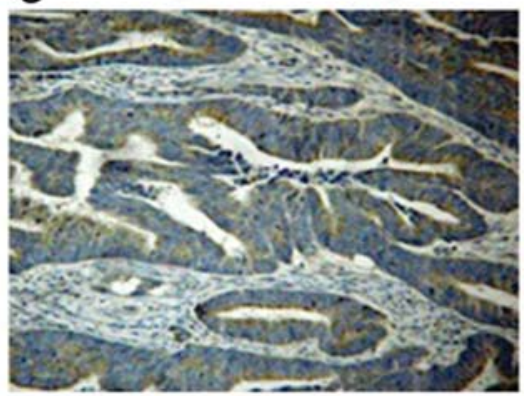

Figure 1. PTEN expression is decreased during human colorectal cancer progression and metastasis. (A) Normal colorectal mucosa, (B) polyps, (C) adenoma, (D) primary colorectal adenocarcinoma Dukes' A, (E) primary colorectal adenocarcinoma Dukes' B, (F) primary colorectal adenocarcinoma Dukes' C, and (G) primary colorectal adenocarcinoma Dukes' D.

3 points, respectively. The number of positive cells was scored as $1, \leq 25 \% ; 2,26-50 \% ; 3,51-75 \%$; and $4, \geq 75 \%$. The total scores were obtained by multiplying the score of the color staining and the point score for the percentage of positive staining. A score of 0 was considered negative. A total score from 1 to 3 points was considered weakly positive. A total score from 4 to 5 points was considered moderately positive. More than 5 points was considered strongly positive.

Measurement of cell proliferation using CCK-8. LoVo, SW480, LoVo pc-DNA3.1, LoVo PTEN, SW480 pc-DNA3.1 and SW480 PTEN cells were seeded in a 96-well plate at 4000 cells/well and cultured in complete RPMI-1640 medium. CCK- 8 was added at 4, 24, 48, 72 and $96 \mathrm{~h}$. The absorbance at 450 and $650 \mathrm{~nm}$ was measured.

Fluorescence-activated cell sorting (FACS). Cells were released using $0.25 \%$ trypsin without EDTA and fixed with $70 \%$ ethanol at $-20^{\circ} \mathrm{C}$ for $12 \mathrm{~h}$. The cells were then washed and incubated with RNase for $30 \mathrm{~min}$ at $37^{\circ} \mathrm{C}$. After PI staining, the cell cycle distribution was assessed using flow cytometry.

For apoptosis analysis, the released LoVo, SW480, LoVo pc-DNA3.1, LoVo PTEN, SW480pc-DNA3.1 and SW480
PTEN cells were stained with Annexin V-FITC and propidium iodide for $15 \mathrm{~min}$ at room temperature. Apoptotic cells were quantified using FACS.

Statistical analysis. All data are presented as the mean \pm standard deviation (SD). Statistical analysis was performed using SPSS13.0 software. A Kruskal-Wallis test was used to analyze the semi-quantitative immunohistochemistry data. Data between two groups were compared using a t-test. One-way ANOVA was used to compare multiple groups. A value of $p<0.05$ was considered to indicate a statistically significant result.

\section{Results}

PTEN expression is decreased during human CRC progression and metastasis. Immunohistochemical staining indicated that PTEN expression was mainly localized in the cytoplasm of colonic epithelial cells. In the normal colorectal mucosa, PTEN staining was negative, weakly positive, positive and strongly positive in $0.00,2.63,18.4$ and $78.9 \%$ of the cells, respectively (Fig. 1A). In colon hyperplastic polyps, PTEN staining was negative, weakly positive, positive and strongly 
Table I. Relationship between PTEN expression and clinical pathology features in the patients with colorectal carcinoma.

\begin{tabular}{|c|c|c|c|c|c|c|c|}
\hline \multirow[b]{2}{*}{ Features } & \multirow[b]{2}{*}{$\mathrm{N}$} & \multicolumn{4}{|c|}{ PTEN expression } & \multirow{2}{*}{$\begin{array}{c}\text { Mean } \\
\text { rank }\end{array}$} & \multirow[b]{2}{*}{ P-value } \\
\hline & & 0 & $1+$ & $2+$ & $3+$ & & \\
\hline \multicolumn{8}{|l|}{ Differentiation } \\
\hline Low & 18 & 12 & 5 & 1 & 0 & 17.92 & 0.052 \\
\hline Moderate & 10 & 5 & 2 & 2 & 1 & 23.90 & \\
\hline Well & 17 & 5 & 6 & 5 & 1 & 27.85 & \\
\hline \multicolumn{8}{|l|}{ Tissue type } \\
\hline Normal mucosa & 38 & 0 & 1 & 7 & 30 & 83.72 & 0.000 \\
\hline Polyp & 19 & 0 & 1 & 2 & 16 & 85.34 & \\
\hline Adenoma & 20 & 5 & 1 & 2 & 12 & 66.08 & \\
\hline Primary tumor & 45 & 22 & 13 & 8 & 2 & 30.63 & \\
\hline \multicolumn{8}{|l|}{ Dukes' stage } \\
\hline A & 6 & 2 & 1 & 2 & 1 & 29.25 & 0.366 \\
\hline $\mathrm{B}$ & 13 & 5 & 5 & 2 & 1 & 25.08 & \\
\hline $\mathrm{C}$ & 20 & 11 & 6 & 3 & 0 & 20.95 & \\
\hline $\mathrm{D}$ & 6 & 4 & 1 & 1 & 0 & 19.08 & \\
\hline
\end{tabular}

positive in $0.00,5.26,10.5$ and $84.29 \%$ of the cells, respectively (Fig. 1B). In colorectal adenomas, PTEN staining was negative, weakly positive, positive and strongly positive in $25,5,10$ and $60 \%$ of the cells, respectively (Fig. 1C). In the CRC tissues, PTEN staining was negative, weakly positive, positive and strongly positive in $48.89,28.89,17.78$ and $4.44 \%$ of the cells, respectively (Fig. 1D-G). As shown in Table I, a significant difference in PTEN expression was found between normal colorectal mucosa, colorectal hyperplastic polyps, colorectal adenomas, and CRC $(\mathrm{P}<0.05$, Kruskal-Wallis test). PTEN expression levels were gradually decreased from normal colorectal mucosa to colorectal hyperplastic polyps to colorectal adenoma and to CRC. PTEN staining was negative, weakly positive, positive and strongly positive in 33.3, 16.67, 33.3 and $16.67 \%$ of the cells, respectively, in Dukes' A colorectal cancer. PTEN staining was negative, weakly positive, positive and strongly positive in $38.46,38.46,15.38$ and $7.69 \%$ of the cells, respectively in Dukes' B colorectal cancer. PTEN staining was negative, weakly positive, positive and strongly positive in 55, 30, 15 and $0.00 \%$ of the cells, respectively in Dukes' $\mathrm{C}$ colorectal cancer. PTEN staining was negative, weakly positive, positive and strongly positive in $66.67,16.67,16.67$ and $0.00 \%$ of the cells, respectively, in Dukes' D colorectal cancer. Although PTEN expression levels differed in Dukes' stage colorectal cancer, no significant difference was found $(\mathrm{P}=0.336)$ due to the small number of samples collected (Table I). It is difficult to collect Dukes' A and Dukes' D colorectal cancers. Therefore, no significant difference in PTEN expression was drawn among CRCs of different grades of differentiation $(\mathrm{P}=0.052)$ (Table I).

Upregulation of PTEN inhibits the proliferation of colon cancer cells. Cell proliferation was detected using CCK-8. Proliferation of LoVo cells transfected with pc-DNA3.1-PTEN was significantly decreased at 24, 48, 72 and 96 h compared
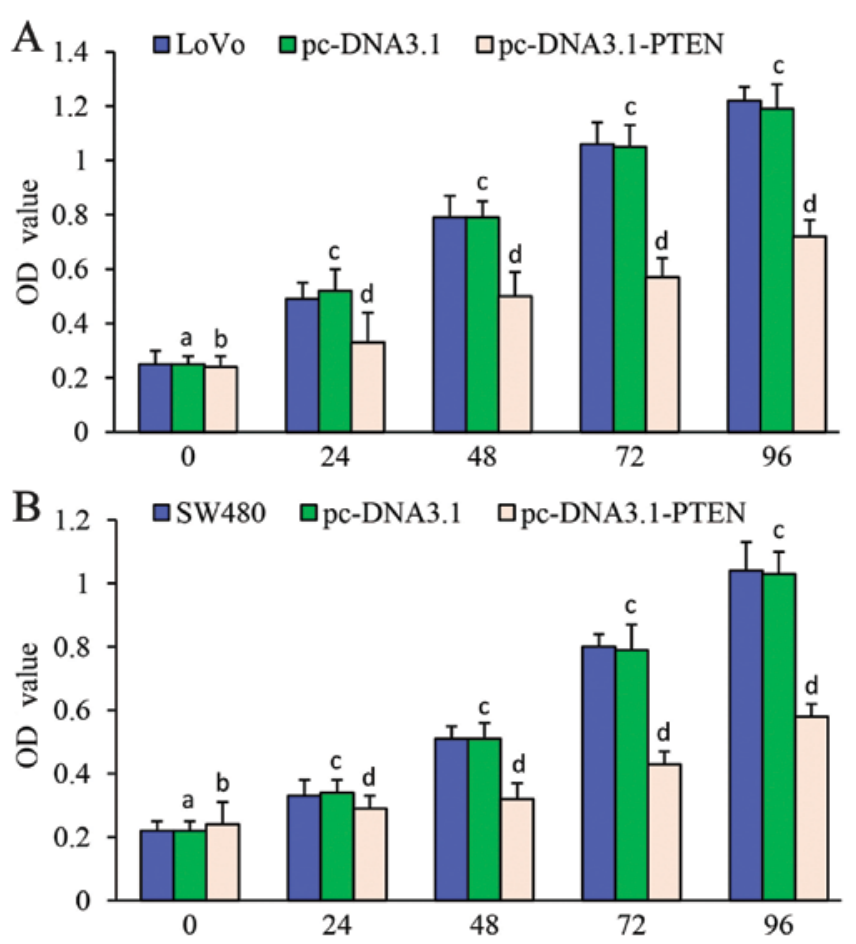

Figure 2. Effect of the upregulation of PTEN on the proliferation of LoVo and SW480 cells. (A) ${ }^{a} \mathrm{P}>0.05$ vs. $\mathrm{LoV}$; ${ }^{\mathrm{b}} \mathrm{P}>0.05$ vs. $\mathrm{LoVo}$ and LoVo pc-DNA3.1; ${ }^{\mathrm{C}} \mathrm{P}>0.05$ vs. LoVo; ${ }^{\mathrm{d}} \mathrm{P}<0.05$ vs. LoVo and LoVo pc-DNA3.1. (B) ${ }^{\mathrm{a}} \mathrm{P}>0.05$ vs. SW480; ${ }^{b} \mathrm{P}>0.05$ vs. SW480 and SW480pc-DNA3.1; ${ }^{\mathrm{C}} \mathrm{P}>0.05$ vs. SW480; ${ }^{\mathrm{d}} \mathrm{P}<0.05$ vs. SW480 and SW480pc-DNA3.1.

with the LoVo cells alone or LoVo cells transfected with pc-DNA3.1. Proliferation rates of the LoVo cells alone and the LoVo cells transfected with pc-DNA3.1 were not significantly different; $(\mathrm{P}>0.05)$ (Fig. 2A). Proliferation of the SW480 cells transfected with pc-DNA3.1-PTEN was significantly decreased at $24,48,72$ and $96 \mathrm{~h}$ compared with the SW480 cells alone and the SW480 cells transfected with pc-DNA3.1 (24 h: F=7.092, $\mathrm{P}=0.002 ; 48$ h: $\mathrm{F}=88.731, \mathrm{P}<0.001 ; 72$ h: $\mathrm{F}=205.396, \mathrm{P}<0.001$; 96 h: $\mathrm{F}=202.248, \mathrm{P}<0.001)$. Proliferation rates of the SW480 cells alone and the SW480 cells transfected with pc-DNA3.1 were not significantly different; ( $\mathrm{P}>0.05)$ (Fig. 2B). These data revealed that upregulation of PTEN expression significantly inhibited the proliferation of the malignant cells.

Upregulation of PTEN blocks cell cycle progression. Compared to the LoVo cells and the LoVo cells transfected with pc-DNA3.1, G1 phase arrest was significantly increased in the LoVo cells transfected with pc-DNA3.1-PTEN ( $\mathrm{F}=15.695$, $\mathrm{P}<0.001$ ) (Fig. 3A). However, the number of cells observed in the $S$ phase was significantly decreased in the LoVo cells transfected with pc-DNA3.1-PTEN (F=28.116, P<0.001) (Fig. 3B). No differences were observed in the cell cycle between the LoVo cells and the LoVo cells transfected with pc-DNA3.1 (P>0.05) (Fig. 3). Similarly, the number of cells observed in the $\mathrm{G} 1$ phase was significantly increased $(\mathrm{F}=25.185, \mathrm{P}<0.001)$ (Fig. 3A), whereas the number of $\mathrm{S}$ phase cells was significantly decreased $(\mathrm{F}=35.199, \mathrm{P}<0.001)$ (Fig. 3B) in the $\mathrm{SW} 480$ cells transfected with pc-DNA3.1-PTEN compared to the SW480 and SW480 cells transfected with pc-DNA3.1. No significant difference was found in the cell cycle between the SW480 cells and the SW480 cells transfected with pc-DNA3.1 

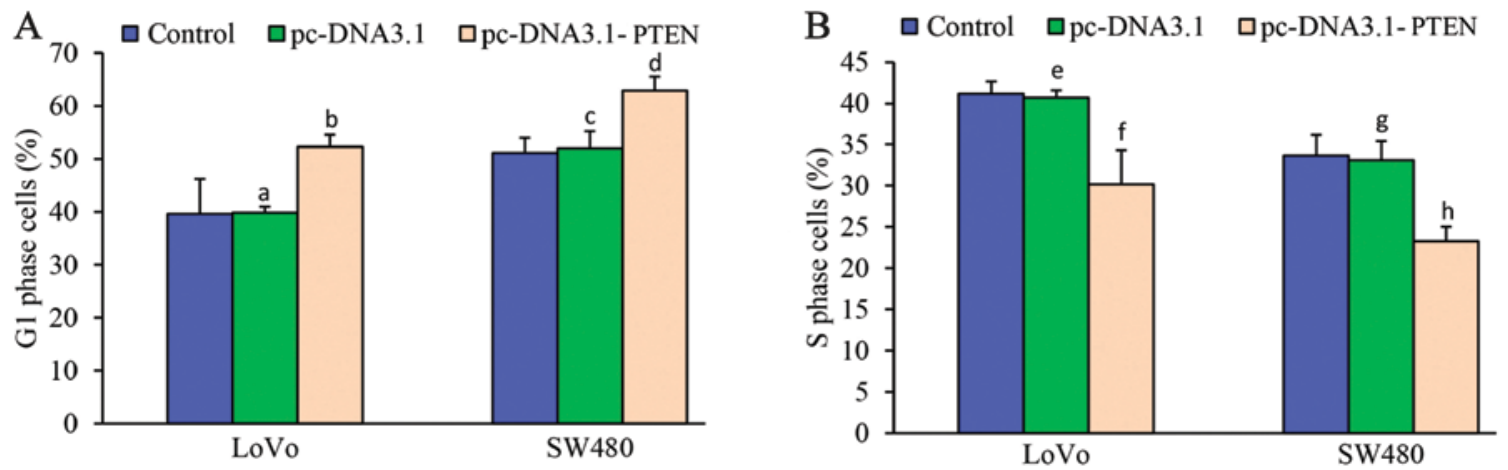

Figure 3. Effect of PTEN expression on the cell cycle distribution in colorectal cancer cells. (A) ${ }^{a} \mathrm{P}>0.05$ vs. LoVo; ${ }^{\mathrm{b}} \mathrm{P}<0.05$ vs. LoVo and LoVo pc-DNA3.1; (B) ${ }^{\mathrm{C}} \mathrm{P}>0.05$ vs. SW480; ${ }^{\mathrm{d}} \mathrm{P}<0.05$ vs. SW480 and SW480pc-DNA3.1; ${ }^{\mathrm{P}}>0.05$ vs. LoVo; ${ }^{\mathrm{f}} \mathrm{P}<0.05$ vs. LoVo and LoVo pc-DNA3.1; ${ }^{\mathrm{g} P}>0.05$ vs. SW480; h, P $<0.05$ vs. SW480 and SW480pc-DNA3.1.
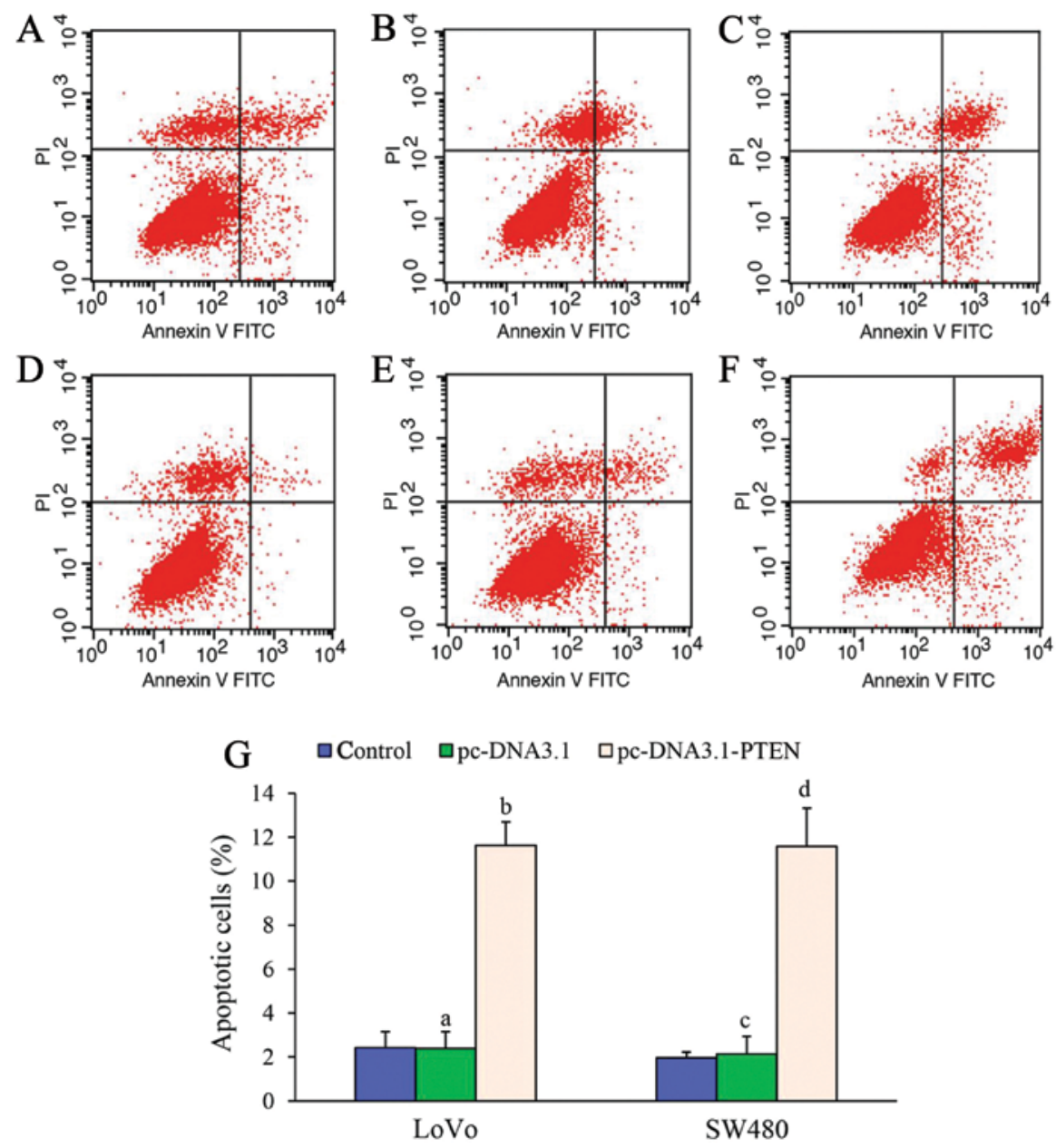

Figure 4. Apoptosis as detected by flow cytometry. (A) LoVo, (B) LoVo pc-DNA3.1, (C) LoVo pc-DNA3.1-PTEN, (D) SW480, (E) SW480 pc-DNA3.1, (F) SW480 pc-DNA3.1-PTEN cells. (G) The percentage of apoptotic cells in the different cell groups.

( $\mathrm{P}>0.05)$ (Fig. 3A and B). These data revealed that upregulation of PTEN blocked G1 phase entry into the S phase, which further inhibited the proliferation of malignant cells.

Upregulation of PTEN increases 5-FU-induced apoptosis in colon cancer cells. Apoptosis was not observed in the LoVo cells or the SW480 cells at $48 \mathrm{~h}$ without 5-FU treatment. As shown in Fig. 4, after cells were treated with $0.01 \mu \mathrm{mol} / \mathrm{ml}$ or $0.03 \mu \mathrm{mol} / \mathrm{ml} 5-\mathrm{FU}$, a significant increase in apoptosis was observed in both the LoVo cells and the SW480 cells transfected with pc-DNA3.1-PTEN compared to the cells alone or cells transfected with pc-DNA3.1. At least a 5-fold increase in apoptosis was found in both the LoVo cells and the SW480 cells transfected with pc-DNA3.1-PTEN compared to the control cells (LoVo: $\mathrm{F}=189.955, \mathrm{P}<0.001$; SW480: $\mathrm{F}=121.719$, $\mathrm{P}<0.001$ ) (Fig. 4). These results suggest that upregulation of 


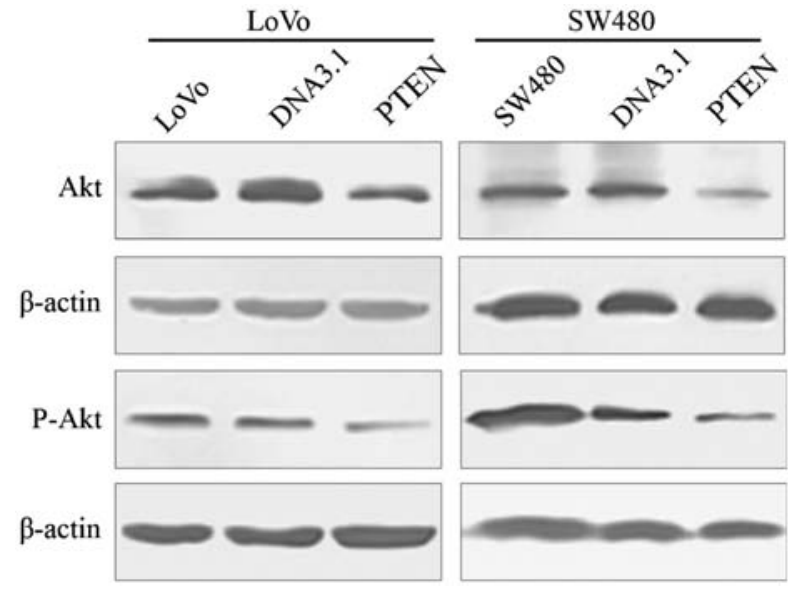

Figure 5. Effect of PTEN expression on Akt and p-Akt.

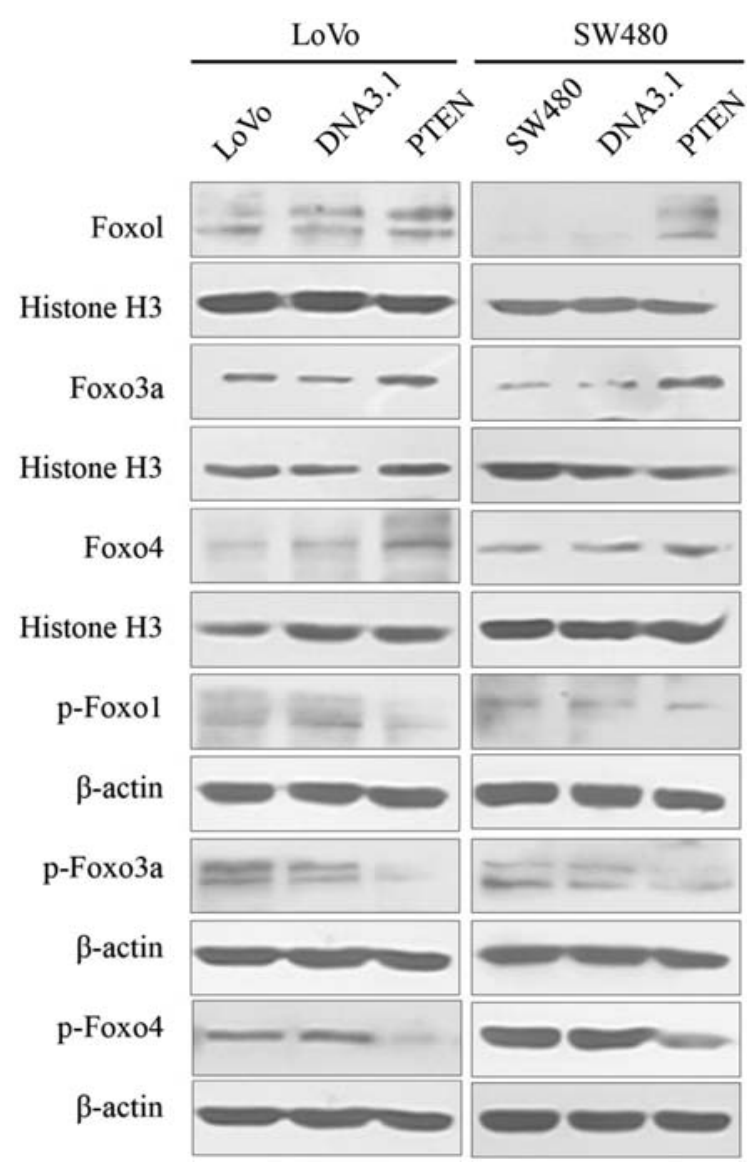

Figure 6. Effect of the upregulation of PTEN on FoxO transcription factors.

PTEN could increase apoptosis in colon cancer cells treated with chemotherapeutic drugs, such as 5-FU.

Effects of upregulation of PTEN on the PI3K/AKT signaling pathway. As shown in Fig. 5, Akt and p-Akt protein expression levels were significantly reduced in both the LoVo cells and the SW480 cells transfected with pc-DNA3.1-PTEN compared to their respective control cells (LoVo: Akt, $\mathrm{F}=420.337, \mathrm{P}<0.001$; p-Akt, $\mathrm{F}=262.269, \mathrm{P}<0.001$ and SW480: Akt, $\mathrm{F}=2300.660$, $\mathrm{P}<0.001$; p-Akt, $\mathrm{F}=109.584, \mathrm{P}<0.001)$. No significant change was found in the LoVo or the SW480 cells compared to

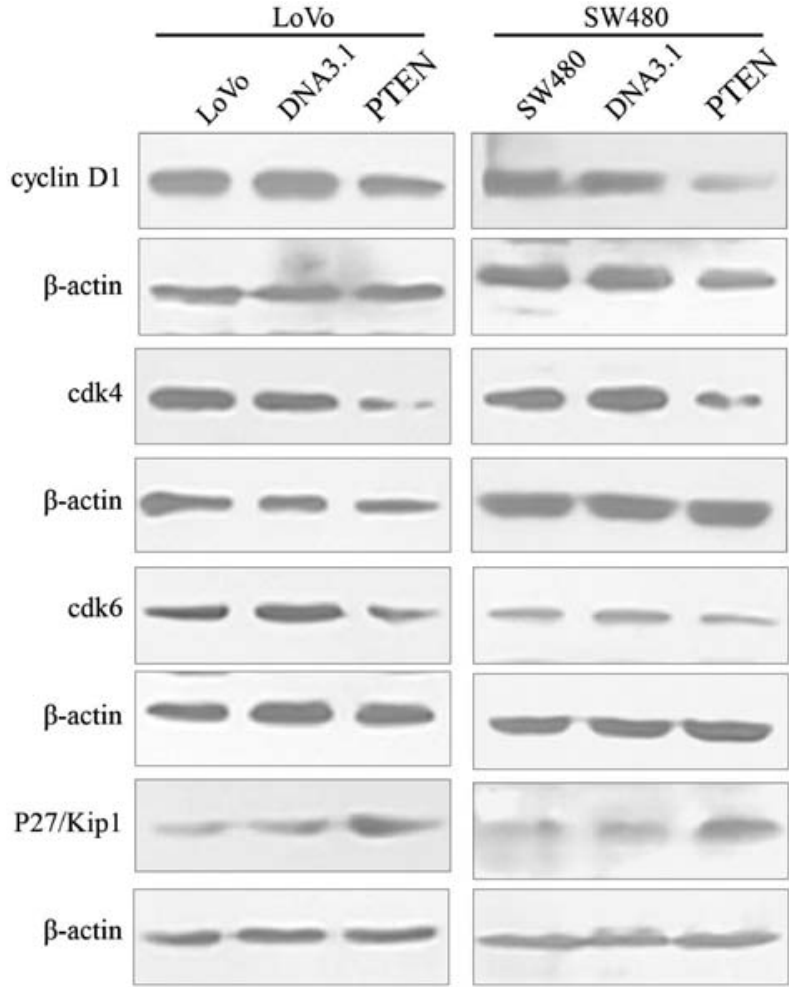

Figure 7. Effect of PTEN expression on cell cycle-associated proteins cyclinD1, cdk4, cdk6 and cyclin kinase inhibitor p27Kip1.

these cells transfected with pc-DNA3.1 ( $\mathrm{P}>0.05)$. These data revealed that upregulation of PTEN protein reduced the expression of Akt.

Nuclear proteins FoxO1 (FKHR), FoxO3a (FKHRL1), and FoxO4 (AFX) were significantly increased in both the LoVo and SW480 cells transfected with pc-DNA3.1-PTEN compared to their respective control cells (LoVo: FoxO1, $\mathrm{F}=37.414$, $\mathrm{P}<0.001$; FoxO3a, $\mathrm{F}=117.618, \mathrm{P}<0.001$; FoxO4, $\mathrm{F}=392.631$, $\mathrm{P}<0.001$ and SW480: FoxO1, $\mathrm{F}=200.868, \mathrm{P}<0.001 ; \mathrm{FoxO} 3 \mathrm{a}$, $\mathrm{F}=2837.989, \mathrm{P}<0.001$; FoxO4, $\mathrm{F}=41.280, \mathrm{P}<0.001$ ) (Fig. 6). Moreover, cytosolic phosphoproteins p-FoxO1, p-FoxO3a, and p-FoxO4 were significantly decreased in both the LoVo and SW480 cells transfected with pc-DNAA3.1-PTEN compared to their respective control cells (LoVo: p-FoxO1, $F=104.490$, $\mathrm{P}<0.001$; p-FoxO3a, $\mathrm{F}=382.393, \mathrm{P}<0.001$; $\mathrm{p}-\mathrm{FoxO} 4, \mathrm{~F}=52.316$, $\mathrm{P}<0.001$ and SW480: $\mathrm{p}-\mathrm{FoxO}, \mathrm{F}=53.754, \mathrm{P}<0.001$; $\mathrm{p}-\mathrm{FoxO} 3 \mathrm{a}$, $\mathrm{F}=39.003, \mathrm{P}<0.001 ; \mathrm{p}-\mathrm{FoxO} 4, \mathrm{~F}=454.873, \mathrm{P}<0.001)$. No significant changes were found between cells alone and cells transfected with pc-DNA3.1.

Cell cycle-related proteins, including cyclinD1, cdk4 and cdk6 were significantly reduced in the both the LoVo and SW480 cells transfected with pc-DNA3.1-PTEN compared to their respective control cells (LoVo: cyclin D1, $\mathrm{F}=151.738$, $\mathrm{P}<0.001 ; \operatorname{cdk} 4, \mathrm{~F}=177.511, \mathrm{P}<0.001 ; \operatorname{cdk6}: \mathrm{F}=1661.422$, $\mathrm{P}<0.001$ and SW480: cyclin D1, $\mathrm{F}=848.596, \mathrm{P}<0.001$; cdk4, $\mathrm{F}=1228.556, \mathrm{P}<0.001$; cdk6, $\mathrm{F}=99.949$, $\mathrm{P}<0.001)$. Cyclindependent kinase inhibitor p27Kip1 was significantly increased in both the LoVo and SW480 cells transfected with pc-DNA3.1-PTEN compared to their respective control cells (LoVo: $\mathrm{F}=854.323, \mathrm{P}<0.001$ and SW480: $\mathrm{F}=140.832$, $\mathrm{P}<0.001)$. No significant changes in cyclinD1, cdk4, cdk6, 
or cyclin-dependent kinase inhibitor p27Kip1 were found between the cells alone and the cells transfected with pc-DNA3.1 (P>0.05) (Fig. 7).

\section{Discussion}

PTEN expression and the progression and metastasis of colorectal cancers (CRCs). CRC is a common malignant cancer. The onset and progression of CRCs are closely related to activation of oncogenes, inactivation of tumor-suppressor genes, gene rearrangement, as well as cell proliferation and apoptosis. Inactivation of tumor-suppressor genes is a very common phenomenon in the progression of CRC, and therefore these proteins are considered as potential molecular targets for CRC treatment.

In 1974, Morson (11) proposed that CRC originated from colorectal adenoma. Research has also suggested that CRC is formed stepwise from normal colon mucosa becoming colorectal adenomas, then adenomas with atypical hyperplasia, and finally carcinoma (12-14). Progression from adenoma to carcinoma generally takes 5-10 years or even longer, and different genes are involved in the formation of carcinoma.

PTEN is a newly discovered tumor-suppressor gene with phosphatase activity. PTEN protein acts as a phosphatase to dephosphorylate phosphatidylinositol $(3,4,5)$-trisphosphate. PTEN specifically catalyzes the dephosphorylation of the 3 ' phosphate of the inositol ring in PIP3, resulting in the biphosphate product PIP2, and inhibiting the phosphorylation of PI3 kinase, thereby blocking Akt and its downstream kinase activity and inducing apoptosis (15).

In order to investigate the relationship between PTEN expression and the progression of CRC, different tissues including normal colon mucosa, colorectal adenomas, adenomas with atypical hyperplasia, and carcinomas were collected to detect PTEN expression using immunohistochemical staining. Our data revealed that PTEN was mainly localized in the cytoplasm of the colorectal epithelial cells and its expression levels in the normal colorectal mucosa and hyperplastic polyps were higher than that in the adenomas. PTEN expression in the CRC tissues was extremely low (Fig. 1, Table I). This suggests that PTEN may play an important role in the evolution of CRC. Of note, downregulation of PTEN was found in adenomas, suggesting that PTEN is involved in an early event during transformation and might be a useful marker for the early diagnosis of CRC. Although a decreasing trend in PTEN expression was observed through different Dukes' stages, this difference was not statistically significant due to the low number of specimens for Dukes' A and Dukes' D stages. In conclusion, these results strongly suggest that PTEN expression might be used as a marker for early diagnosis of CRC and may provide a new target for cancer treatment.

Effects of the upregulation of PTEN on proliferation and apoptosis in CRC cells. Cell proliferation, differentiation and apoptosis are essential to maintain cell homeostasis. The PI3K/Akt pathway is closely associated with tumor development. The tumor-suppressor gene PTEN is highly expressed in a variety of normal human tissues, such as placenta, kidney, liver and brain. Mutations in the PTEN gene are closely related to cancer-predisposing genetic diseases including Cowden's BRRS syndrome (16). PTEN mutations have been found in a variety of tumors including prostate cancer, endometrial cancer, glioma, lung cancer and liver cancer (17).

In the present study, in order to investigate whether upregulation of PTEN affects the cell cycle, proliferation and apoptosis, PTEN was overexpressed in LoVo and SW480 CRC cell lines. Our data indicated that the proliferation of LoVo and SW480 cells transfected with pc-DNA3.1-PTEN was significantly decreased when compared to the control cells. These data revealed that upregulation of PTEN could inhibit the proliferation of tumor cells. Moreover, significant increases were observed in the number of cells in the G1 phase, while the number of cells in the $S$ phase was decreased in the LoVo and SW480 cells transfected with pc-DNA3.1-PTEN compared to the control cells. This indicated that upregulation of PTEN could inhibit cell proliferation though blockage of G1/S phase transition.

When these cells were treated with 5-FU, increased apoptosis was observed in the LoVo and SW480 cells transfected with pc-DNA3.1-PTEN compared to the control cells. This indicated that upregulation of PTEN could increase the sensitivity of colon cancer cells to chemotherapeutic drugs such as 5-FU by inducing apoptosis. The mechanism behind PTEN-induced apoptosis is not yet fully understood, but may be related to the PI3K/Akt signaling pathway. Since PTEN can regulate PIP3 levels, this may subsequently affect the PIP3-PKB/Akt apoptotic pathway. Mutations in the PTEN gene in tumor cells could decrease its phosphatase activity, leading to cell proliferation and a reduction in apoptosis (18).

PTEN and the PI3K/Akt/FoxO signaling pathway in CRC. The $\mathrm{PI} 3 \mathrm{~K} / \mathrm{Akt}$ signaling pathway is closely related to tumor onset and tumor development. PI (3-5) P3, the catalytic product of PI3K, can activate downstream signal transduction molecules, inducing tumor proliferation and inhibition of apoptosis. PTEN suppresses tumor formation by restraining the PI3K/ AKT pathway (19). PTEN can inhibit the phosphorylation of PIP3 kinase, blocking Akt and its downstream kinase activity and inducing apoptosis. Mutation of the PTEN gene can lead to abnormal activation of PIP3 and prevention of cell death. In agreement with previous studies, expression levels of Akt and P-Akt were significantly decreased in the Lovo and SW480 cells transfected with pc-DNA3.1-PTEN compared to the control cells.

FoxO, a subgroup of the forkhead family, is a transcription factor that plays an important role in regulating the expression of genes involved in cell growth, proliferation and apoptosis. The phosphorylation of FoxO is necessary for its activity and is regulated by a variety of kinases (20). The protein kinase Akt can regulate phosphorylation of FoxO at serine and threonine residues (T1, T2, S1, S2 and S3), and inhibit FoxO transcriptional activity (21). FoxO is mainly localized in the nucleus, but Akt-mediated phosphorylation of FoxO induces FoxO transportation to the cytoplasm $(21,22)$. Inhibition of $\mathrm{Akt} / \mathrm{PKB}$ retains FoxO in the nucleus, whereas activation of $\mathrm{PKB} / \mathrm{Akt}$ releases FoxO to the cytoplasm and leads to inhibition of gene transcription (23-25).

Medema et al (26) reported that infection of mouse embryonic fibroblasts (MEFs) with Foxo4 retrovirus could induce 
the expression of P27kip (cyclin-dependent kinase inhibitor, CDKI) and increase P27kip1 and cyclin E/cdk2 complex formation, causing G1 phase arrest. Kops et al (27) reported that infection of NIH $3 \mathrm{~T} 3$ mouse fibroblasts and MEFs with FoxO4 and FoxO3 (FKHR-L1) retroviruses induced expression of retinoblastoma-like 130 protein $\mathrm{Rb}$, which forms a complex with E2F-4 and reduces the level of free E2F-4 in the nucleus to inhibit cell cycle progression. With the exception of CDK, FoxO regulates expression of cyclins, including cyclins A, B, D, E, G, and $\mathrm{H}$ and inhibits cell cycle progression. FoxO factors can inhibit expression of cyclins D1 and D2, and Foxo3a can elevate the expression of cyclin G2 $(28,29)$. In other words, FoxO proteins play important roles in the onset and progression of tumors by regulating the cell cycle and apoptosis. Mutations or decreases in FoxO can inhibit apoptosis and promote cell overgrowth, leading to tumor expansion (9).

The inhibition of G1-S phase transition by PTEN is dependent on Akt/PKB and can be restored by active Akt/PKB (30). Overexpression of PTEN increases the expression of P27kip and P27kip1/cyclin E/cdk2 complex formation and decreases cyclin D3 levels and CDK2 kinase activity, eventually halting the cell cycle at the $\mathrm{G} 1$ phase $(18,31,32)$. Moreover, PTEN can overcome the phosphorylation of glycogen synthase kinase 3 (GSK3) by Akt/PKB. The phosphorylation of GSK3 promotes degradation of cyclinD1 and arrests the cell cycle and inhibits cell growth (33). Wu et al found that PTEN can induce three cyclin-dependent kinase inhibitors, P21waf1, P27kip1 and P57 kip1, thereby regulating the cell cycle (34). Research has indicated that PI3-K/Akt can overcome NF- $\kappa$ B and CD95/Fasinduced apoptosis, while PTEN can inhibit TNF-mediated $\mathrm{NF}-\kappa \mathrm{B}$ activity $(35,36)$. PTEN can also induce apoptosis by activating caspase-3, caspase- 8 and FADD-dependent pathways. Overexpression of PTEN in SHG-44 glioma cells can induce apoptosis (37). It has been reported that PTEN can activate the P53 signaling pathway in glioma cells, negatively regulating cell division and proliferation (38). In turn P53 can induce PTEN expression and cell apoptosis $(39,40)$.

In the present study, we first addressed whether upregulation of PTEN activates the downstream transcription factor FoxO. Our data indicated that nuclear FoxO1 (FKHR), FoxO3a (FKHRL1) and FoxO4 (AFX) were significantly increased in both LoVo cells and SW480 cells transfected with pc-DNA3.1-PTEN, while cytosolic p-FoxO1 (FKHR, $\mathrm{Ser}^{256}$ ), p-FoxO3a (FKHRL1, Ser $^{253}$ ) and p-FoxO4 (AFX, Ser ${ }^{193}$ ) were significantly decreased. PTEN overexpression induced FoxO transportation from the nucleus to the cytoplasm and promoted the transcription of downstream genes involved in cell proliferation and apoptosis. Levels of cell cycle-related proteins cyclinD1, cdk4 and cdk6 were significantly decreased in the LoVo and SW480 cells transfected with pc-DNA3.1PTEN, while cyclin-dependent kinase inhibitor p27/Kip1 expression was increased significantly. These results revealed that upregulation of PTEN protein reduced Akt activity and phosphorylated FoxO, which further inhibited cell cycle progression. This further confirmed that upregulation of PTEN can inhibit CRC cell proliferation and normal cell cycle progression. Reagan-Shaw and Ahmad (41) showed that RNAi against PI3K in breast cancer cells enhanced FoxO activation and eliminated the inhibition of FoxO-mediated cell growth and apoptosis. Upregulation of PTEN can induce the apoptosis of colon cancer cells treated with 5-FU, which may be related to FoxO activity.

In conclusion, this is the first report of a gradual decrease in expression of PTEN from normal colon epithelial tissues to colon hyperplastic polyps, colorectal adenomas and finally CRC. Overexpression of PTEN in CRC cell lines inhibited cell proliferation and cell cycle progression and increased 5-FU-induced apoptosis. Upregulation of PTEN inhibited the activity of the Akt pathway and regulated downstream genes involved in the cell cycle. This suggests that inhibition of CRC cell proliferation and cell cycle arrest by PTEN is closely related to PI3K/Akt/FoxO. This study elucidated the molecular mechanism behind the malignant proliferation of colon cancer cells and provides a new target for the gene therapy and prognosis of CRC.

\section{Acknowledgements}

The present study was supported by the Science and Information Technology Foundation of Guangzhou (grant nos. 2010J E141 and 2011J4100051), the Guangdong Provincial Science and Technology Department (grant no. 2011B0904005260) and the Natural Science Foundation of Guangdong Province (grant no. S2012040006707).

\section{References}

1. Siegel R, Naishadham D and Jemal A: Cancer statistics, 2012. CA Cancer J Clin 62: 10-29, 2012.

2. You WC, Jin F, Devesa S, et al: Rapid increase in colorectal cancer rates in urban Shanghai, 1972-97, in relation to dietary changes. J Cancer Epidemiol Prev 7: 143-146, 2002.

3. Cerottini JP, Caplin S, Pampallona S and Givel JC: Prognostic factors in colorectal cancer. Oncol Rep 6: 409-414, 1999.

4. Parsons DW, Wang TL, Samuels Y, et al: Colorectal cancer: mutations in a signalling pathway. Nature 436: 792, 2005.

5. Lee JO, Yang H, Georgescu MM, et al: Crystal structure of the PTEN tumor suppressor: implications for its phosphoinositide phosphatase activity and membrane association. Cell 99: 323-334, 1999.

6. Di Cristofano A and Pandolfi PP: The multiple roles of PTEN in tumor suppression. Cell 100: 387-390, 2000.

7. Cully M, You H, Levine AJ and Mak TW: Beyond PTEN mutations: the PI3K pathway as an integrator of multiple inputs during tumorigenesis. Nat Rev Cancer 6: 184-192, 2006.

8. Accili D and Arden KC: FoxOs at the crossroads of cellular metabolism, differentiation, and transformation. Cell 117: 421-426, 2004.

9. Greer EL and Brunet A: FOXO transcription factors at the interface between longevity and tumor suppression. Oncogene 24: 7410-7425, 2005

10. Brunet A, Bonni A, Zigmond MJ, et al: Akt promotes cell survival by phosphorylating and inhibiting a Forkhead transcription factor. Cell 96: 857-868, 1999.

11. Morson BC: The evolution of colorectal carcinoma. Clin Radiol 35: 425-431, 1984

12. Sherman DW, Ye XY, McSherry C, Parkas V, Calabrese M and Gatto M: Quality of life of patients with advanced cancer and acquired immune deficiency syndrome and their family caregivers. J Palliat Med 9: 948-963, 2006.

13. Zhang SQ: Screening and prevention of colorectal cancer in Haining County. Dis Colon Rectum 28: 300-304, 1985.

14. Zhang SQ, Zhu SX and Wu JM: Screening and prevention of colorectal cancer in Haining county. Chin Med J 93: 843-848, 1980.

15. Stambolic V, Suzuki A, de la Pompa JL, et al: Negative regulation of PKB/Akt-dependent cell survival by the tumor suppressor PTEN. Cell 95: 29-39, 1998.

16. Eng C: PTEN: one gene, many syndromes. Hum Mutat 22: 183-198, 2003. 
17. Rustia A, Wierzbicki V, Marrocco L, Tossini A, Zamponi C and Lista F: Is exon 5 of the PTEN/MMAC1 gene a prognostic marker in anaplastic glioma? Neurosurg Rev 24: 97-102, 2001.

18. Cheney IW, Neuteboom ST, Vaillancourt MT, Ramachandra M and Bookstein R: Adenovirus-mediated gene transfer of $M M A C 1 / P T E N$ to glioblastoma cells inhibits $\mathrm{S}$ phase entry by the recruitment of $\mathrm{p} 27^{\mathrm{Kipl}}$ into cyclin E/CDK2 complexes. Cancer Res 59: 2318-2323, 1999.

19. Cantley LC and Neel BG: New insights into tumor suppression: PTEN suppresses tumor formation by restraining the phosphoinositide 3-kinase/AKT pathway. Proc Natl Acad Sci USA 96: 4240-4245, 1999.

20. Burgering BM and Kops GJ: Cell cycle and death control: long live Forkheads. Trends Biochem Sci 27: 352-360, 2002.

21. Burgering BM and Medema RH: Decisions on life and death: FOXO Forkhead transcription factors are in command when PKB/Akt is off duty. J Leukoc Biol 73: 689-701, 2003.

22. Brownawell AM, Kops GJ, Macara IG and Burgering BM: Inhibition of nuclear import by protein kinase B (Akt) regulates the subcellular distribution and activity of the forkhead transcription factor AFX. Mol Cell Biol 21: 3534-3546, 2001.

23. Shi F and LaPolt PS: Relationship between FoxO1 protein levels and follicular development, atresia, and luteinization in the rat ovary. J Endocrinol 179: 195-203, 2003.

24. Brunet A, Kanai F, Stehn J, et al: 14-3-3 transits to the nucleus and participates in dynamic nucleocytoplasmic transport. J Cell Biol 156: 817-828, 2002.

25. Ramaswamy S, Nakamura N, Sansal I, Bergeron L and Sellers WR: A novel mechanism of gene regulation and tumor suppression by the transcription factor FKHR. Cancer Cell 2: 81-91, 2002.

26. Medema RH, Kops GJ, Bos JL and Burgering BM: AFX-like Forkhead transcription factors mediate cell-cycle regulation by Ras and PKB through p27 $7^{\text {kipl. }}$. Nature 404: 782-787, 2000.

27. Kops GJ, Medema RH, Glassford J, et al: Control of cell cycle exit and entry by protein kinase B-regulated forkhead transcription factors. Mol Cell Biol 22: 2025-2036, 2002.

28. Schmidt M, Fernandez de Mattos S, van der Horst A, et al: Cell cycle inhibition by FoxO forkhead transcription factors involves downregulation of cyclin D. Mol Cell Biol 22: 7842-7852, 2002.

29. Martinez-Gac L, Marques M, Garcia Z, Campanero MR and Carrera AC: Control of cyclin G2 mRNA expression by forkhead transcription factors: novel mechanism for cell cycle control by phosphoinositide 3-kinase and forkhead. Mol Cell Biol 24: 2181-2189, 2004
30. Ramaswamy S, Nakamura N, Vazquez F, et al: Regulation of G1 progression by the PTEN tumor suppressor protein is linked to inhibition of the phosphatidylinositol 3-kinase/Akt pathway. Proc Natl Acad Sci USA 96: 2110-2115, 1999.

31. Di Cristofano A, De Acetis M, Koff A, Cordon-Cardo C and Pandolfi PP: Pten and p27 ${ }^{\mathrm{KIP} 1}$ cooperate in prostate cancer tumor suppression in the mouse. Nat Genet 27: 222-224, 2001.

32. Zhu X, Kwon CH, Schlosshauer PW, Ellenson LH and Baker SJ: PTEN induces G(1) cell cycle arrest and decreases cyclin D3 levels in endometrial carcinoma cells. Cancer Res 61: 4569-4575, 2001.

33. Weng LP, Brown JL and Eng C: PTEN coordinates G(1) arrest by down-regulating cyclin D1 via its protein phosphatase activity and up-regulating p27 via its lipid phosphatase activity in a breast cancer model. Hum Mol Genet 10: 599-604, 2001.

34. Wu RC, Li X and Schonthal AH: Transcriptional activation of p21WAF1 by PTEN/MMAC1 tumor suppressor. Mol Cell Biochem 203: 59-71, 2000.

35. Romashkova JA and Makarov SS: NF-kappaB is a target of AKT in anti-apoptotic PDGF signalling. Nature 401: 86-90, 1999.

36. Gustin JA, Maehama T, Dixon JE and Donner DB: The PTEN tumor suppressor protein inhibits tumor necrosis factor-induced nuclear factor kappa B activity. J Biol Chem 276: 27740-27744, 2001.

37. Yuan XJ and Whang YE: PTEN sensitizes prostate cancer cells to death receptor-mediated and drug-induced apoptosis through a FADD-dependent pathway. Oncogene 21: 319-327, 2002.

38. Huang H, Cheville JC, Pan Y, Roche PC, Schmidt LJ and Tindall DJ: PTEN induces chemosensitivity in PTEN-mutated prostate cancer cells by suppression of Bcl-2 expression. J Biol Chem 276: 38830-38836, 2001.

39. Mayo LD, Dixon JE, Durden DL, Tonks NK and Donner DB: PTEN protects $\mathrm{p} 53$ from $\mathrm{Mdm} 2$ and sensitizes cancer cells to chemotherapy. J Biol Chem 277: 5484-5489, 2002.

40. Stambolic V, MacPherson D, Sas D, et al: Regulation of PTEN transcription by $\mathrm{p} 53$. Mol Cell 8: 317-325, 2001.

41. Reagan-Shaw S and Ahmad N: RNA interference-mediated depletion of phosphoinositide 3-kinase activates forkhead box class $\mathrm{O}$ transcription factors and induces cell cycle arrest and apoptosis in breast carcinoma cells. Cancer Res 66: 1062-1069, 2006.

42. Carter JH, Douglass LE, Deddens JA, et al: Pak-1 expression increases with progression of colorectal carcinomas to metastasis. Clin Cancer Res 10: 3448-3456, 2004. 